\title{
Inclusive and differential top quark pair cross- section measurements at CMS
}

\author{
Javier Fernández Menéndez * \\ Universidad de Oviedo, ICTEA \\ C/ Federico García Lorca s/n, \\ Oviedo, Spain \\ E-mail: javier.fernandez@cern.ch
}

Recent results and state-of-the-art on top-quark pair production cross sections in both inclusive and differential measurements are presented, obtained using data collected with the CMS experiment during the LHC Run2 period at centre-of-mass energies ranging of 5.02 and $13 \mathrm{TeV}$. Results are confronted against SM NLO and NNLO order predictions, several MC generators and tunes, and parton density function variations, with a special focus on the latest Run 2 results.

The European Physical Society Conference on High Energy Physics (EPS_HEP2021) 26-30 July 2021

Online conference, jointly organized by Universität Hamburg and the research center DESY

* Speaker, on behalf of the CMS collaboration 


\section{Introduction}

The enormous datasets provided and the high production rate of top quark pairs $(\mathrm{t} \overline{\mathrm{t}})$ at the LHC allow not only accurate inclusive cross section determinations but also precise measurements of differential and double-differential cross sections as a function of kinematic variables of the top quark and the $t \bar{t}$ system. As the higher order corrections to $t \bar{t}$ production are large, these measurements test the predictive power of perturbative quantum chromodynamics (QCD) calculations and electro-weak corrections. Similarly, $\mathrm{t} \overline{\mathrm{t}}$ observables at particle level can constrain the parton shower and hadronisation modelling. Due to the large mass of the top quark, $t \bar{t}$ cross sections are sensitive to the parton distribution function (PDF) of the gluon. Hence, these measurements have the potential to reduce the PDF uncertainties. Moreover, the generation of $t \bar{t}$ events requires a realistic modeling of parton showers. Measurements of kinematic properties and multiplicities of jets associated with $\mathrm{t} \overline{\mathrm{t}}$ production allow a detailed comparison of different parton shower models with the data and provide insight into their tuning.

Extensive measurements were performed by the CMS [1] experiment at different protonproton center-of-mass energies ranging from 5.02 to $13 \mathrm{TeV}$. This document shows the current latest results and correspond to data taken during the Run2 period up to the year 2018.

\section{Inclusive cross section measurements}

With full next-to-next-to-leading order plus next-to-next-leading logarithmic order $(\mathrm{NNLO}+\mathrm{NNLL})$ [2] predictions available for $\mathrm{t} \overline{\mathrm{t}}$ production, precise measurements of the inclusive cross section are important tests for the underlying theory. The $t \bar{t}$ pair decays allow an event selection based on the topology of the particular decay of the $\mathrm{W}$ bosons as shown in Figure 1. There has been an excellent progress both on the theory and experiment side as shown in Figure 2 , with an impressive agreement on the inclusive cross-section up to now where the experimental uncertainty is comparable to the theoretical one.

A special remark must be made to the latest inclusive additions: the inclusive measurement at $5.02 \mathrm{TeV}$ using the dilepton $\mathrm{e} \mu$ channel and data taken $\left(304 \mathrm{pb}^{-1}\right)$ in 2017 [3], achieving a precision of $7.9 \%$ after the combination with the previous lepton + jets channel analysis with data taken in 2015; the inclusive e $/ \mu+$ jets at $13 \mathrm{TeV}$ (which will be referenced again later in this document for its differential measurements) with full Run2 data [4], which achieves the best precision so far in this kind of measurements (3.2\%); and finally the first $t \bar{t}$ pair measurement at $13 \mathrm{TeV}$ including taus in decays [5], improving the relative precision of previous 7 and $8 \mathrm{TeV}$. These two last results are highlighted in Figure 1 in yellow and blue respectively, while the first result is highlighted in Figure 2 in red.

The impressive agreement up to now of the inclusive measurements across the whole centerof-mass energies range, with experimental uncertainties comparable to the theoretical uncertainty, has triggered the need to look at differential cross section measurements in the search for signs Beyond the Standard Model (SM). The following sections collect the latest results in this matter. 


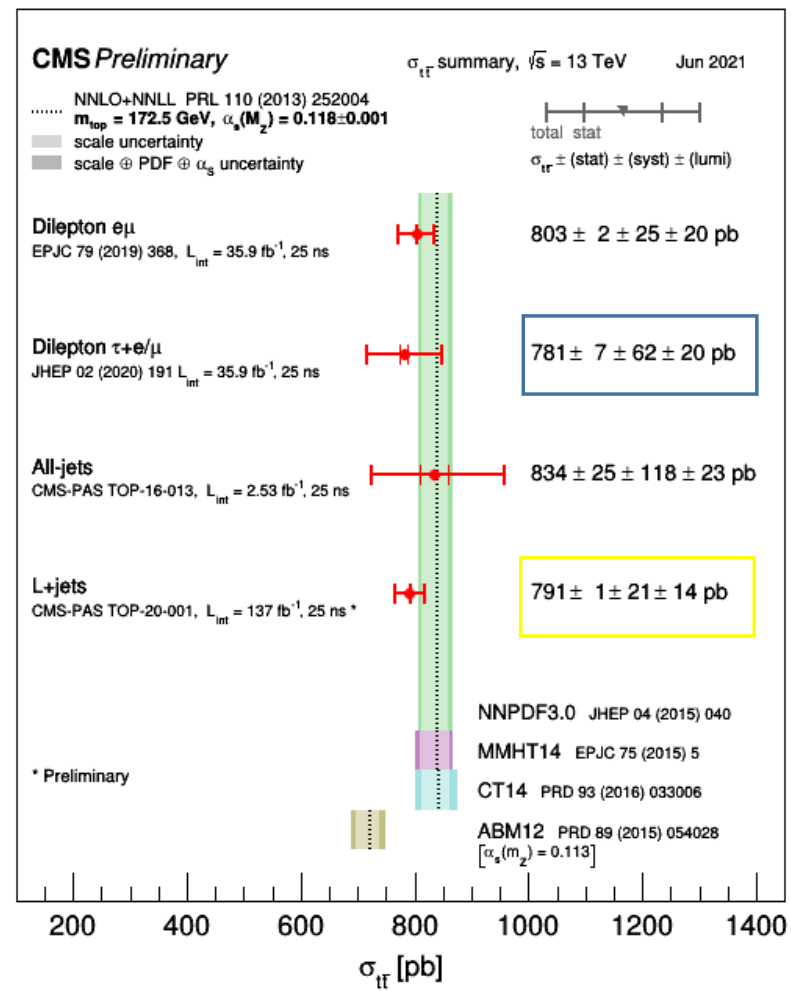

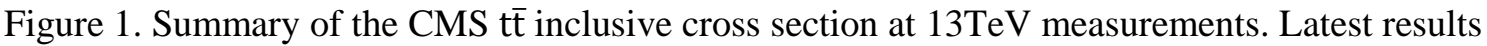
highlighted in yellow and blue.

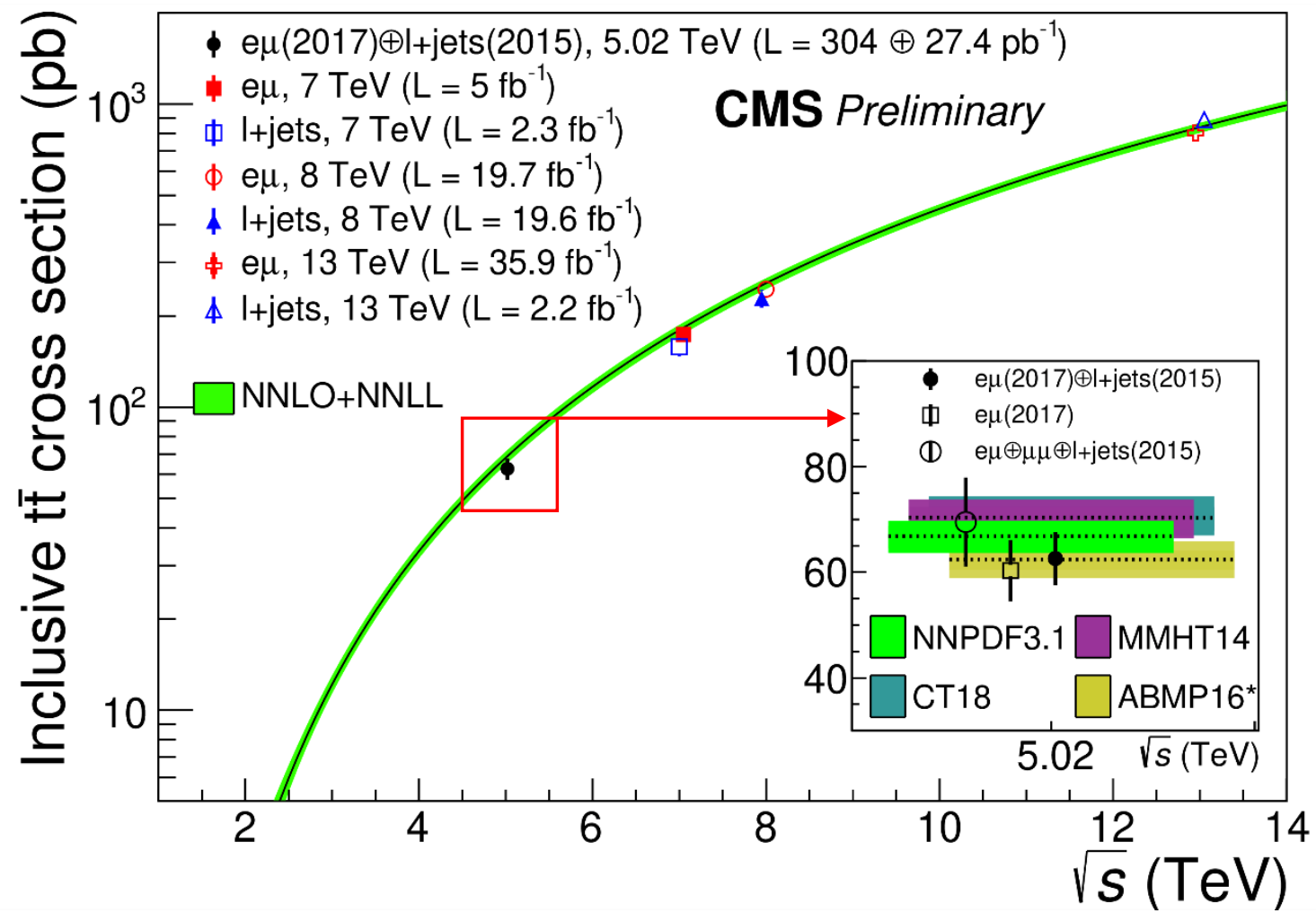

Figure 2. Evolution of the $t \bar{t}$ inclusive cross section measurements with $\sqrt{s}$ in the $1+$ jets and dilepton channels. The vertical bars and bands represent the total uncertainties in the data and in the predictions, respectively. Latest result at 5.02TeV highlighted in yellow and the zoomed box. 


\section{Differential and double cross section measurements.}

Since Run1 there has been an ongoing discrepancy in full next-to-leading order (NLO) modelling of the top and $t \bar{t}$ system, most famously in the top quark transverse momentum $\mathrm{p}_{\mathrm{T}}(\mathrm{t})$. It has been observed in many channels, analyses, fiducial and full phase-space data, in predictions at particle and parton level. New results confirm this discrepancy and provide more precision including even electroweak corrections. The latest boosted analysis from CMS at 13TeV[6], performed in both the hadronic and lepton+jets channel, observes absolute cross sections significantly lower than the predictions from theory, while the normalized differential distributions are well described. Figure 3 shows an example of some notable discrepancies.
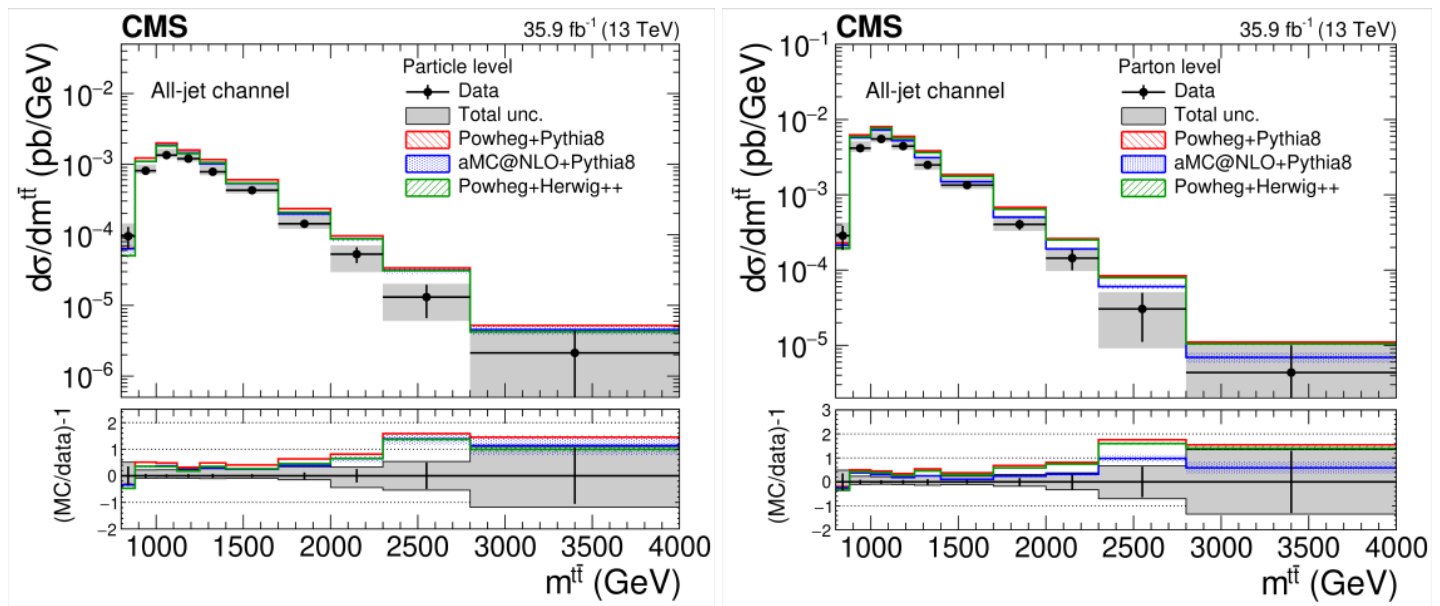

Figure 3. Boosted differential $t \bar{t}$ production cross sections at particle level in fiducial phase space (left) and parton level in full phase space (right) as a function of $m(t \bar{t})$ as seen in [6]. Several full NLO MC setups interfaced with Pythia8 or Herwig++ for Parton Shower (PS) are shown. The lower panel in each plot shows the ratio of the theoretical prediction to the data.

At present the latest differential result [4] using the full Run2 data $\left(137 \mathrm{fb}^{-1}\right)$ and the e/ $\mu+$ jets channel, provides for the first time the cross section of the full spectra obtained using a combination of resolved and boosted $t \bar{t}$ topologies. The combination of multiple reconstruction categories allows for constraints of systematic uncertainties and results in a significantly improved precision with respect to previous measurements . Uncertainties in the jet energy scale, luminosity, and $t \bar{t}$ modeling are the dominant sources.

The four event categories which where combined based of number of tight (loose) b-tags and resolved or boosted top-quark candidates are the following:

- $2 \mathrm{t}: 2$ resolved top-candidates with 2 tightly b-tagged jets

- 1t11: 2 resolved top-candidates with 1 tight and 1 loose b-tag

- BHRL: boosted hadronic top $\left(\mathrm{p}_{\mathrm{T}}>400 \mathrm{GeV}\right)$ but resolved leptonic top, 1 tight $b$-tag

- BHBL: both top-candidates boosted, 1 loose b-tag on leptonic side

Figure 4 shows a distribution of transverse momentum for the hadronic top $\left(\mathrm{p}_{\mathrm{T}}\left(\mathrm{t}_{\mathrm{h}}\right)\right)$ with the 4 categories (left), and the differential cross section after normalization at parton level (right), where it can be apreciated once again how the absolute prediction is above the obtained data but how after normalization shapes come to agreement with the measurement. 

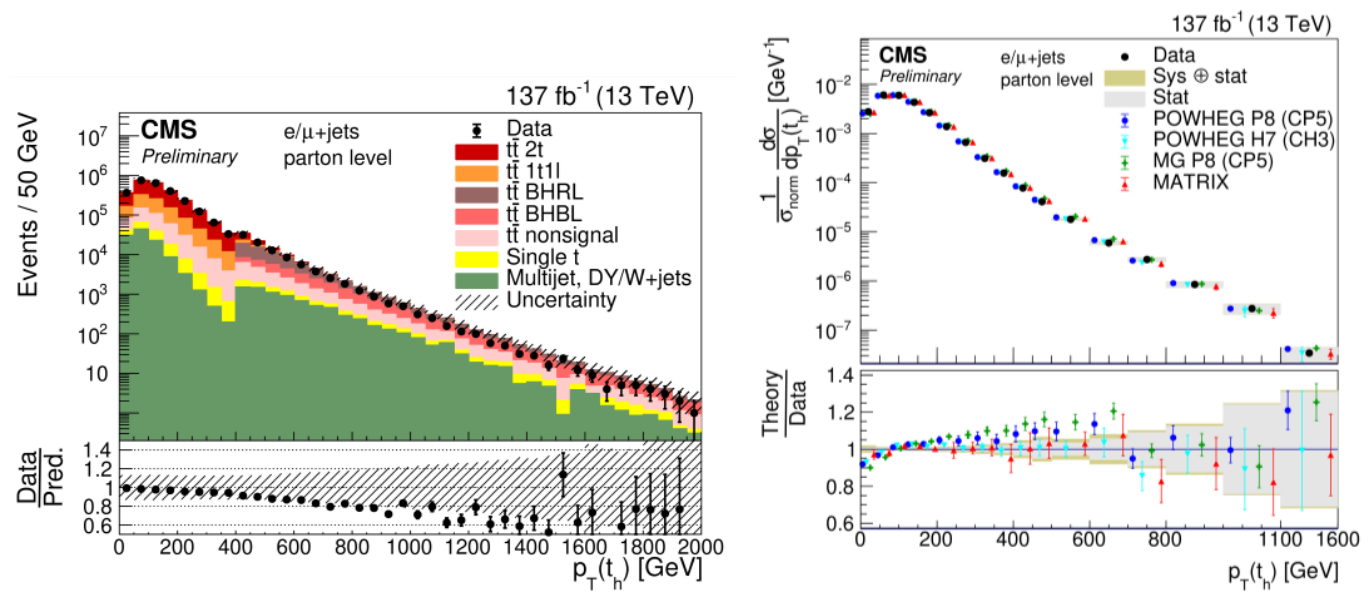

Figure 4. Non-normalized distribution for the different event categories considered (left) and normalized differential $t \bar{t}$ production cross section (right) at parton level as a function of $\mathrm{p}_{\mathrm{T}}\left(\mathrm{t}_{\mathrm{h}}\right)$ as seen in [4]. The data are shown as points with light (dark) bands indicating the statistical (statistical and systematic) uncertainties. The cross sections are compared to the predictions of POWHEG combined with PYTHIA (P8) or HERWIG (H7), the multiparton simulation MG5_aMC@NLO (MG5)+PYTHIA FxFx, and the NNLO QCD calculations obtained with Matrix. The ratios of the various predictions to the measured cross sections (right) and the ratio of measured distribution to nominal Monte Carlo are shown at the bottom of the panel.

The so called 2D or double differential studies provide more details in corners of the phase space while looking at two observables at the same time. The latest results from Run2[4] provide also double differential results with the same analysis premises commented above. Figure 5 shows the result where the overprediction of the $\mathrm{p}_{\mathrm{T}}\left(\mathrm{t}_{\mathrm{h}}\right)$ is clearly seen specially for the 0 additional jets case which is most sensitive to NLO generator effects; the 1 additional jet bin is really probing the NLO part of the calculation with a smaller effect, while 2 or 3 extra jets have increased systematic uncertainties and are most sensitive to parton shower.

\section{Summary and conclusions}

CMS inclusive $t \bar{t}$ cross section results are in good agreement with predictions up to now. However, discrepancies have arisen since Run1 when looking at differential distributions, in particular in the full NLO modelling of observables coming from the top and $t \bar{t}$ systems. A key tool for answering this puzzle are differential 1D/2D cross section measurements, since Run2 at $13 \mathrm{TeV}$ data allows to explore the full phase space of top production in both boosted and resolved topologies.

The differential cross sections are presented at the parton and particle level, where the latter minimizes extrapolations based on theoretical assumptions. Both results are compared to SM calculations using different combinations of NLO QCD matrix elements and parton shower models. Most of the measured differential cross sections are well described by the predictions with the exception of some double-differential distributions. A calculation at NNLO accuracy in QCD provides an improved description of the parton-level measurements. 

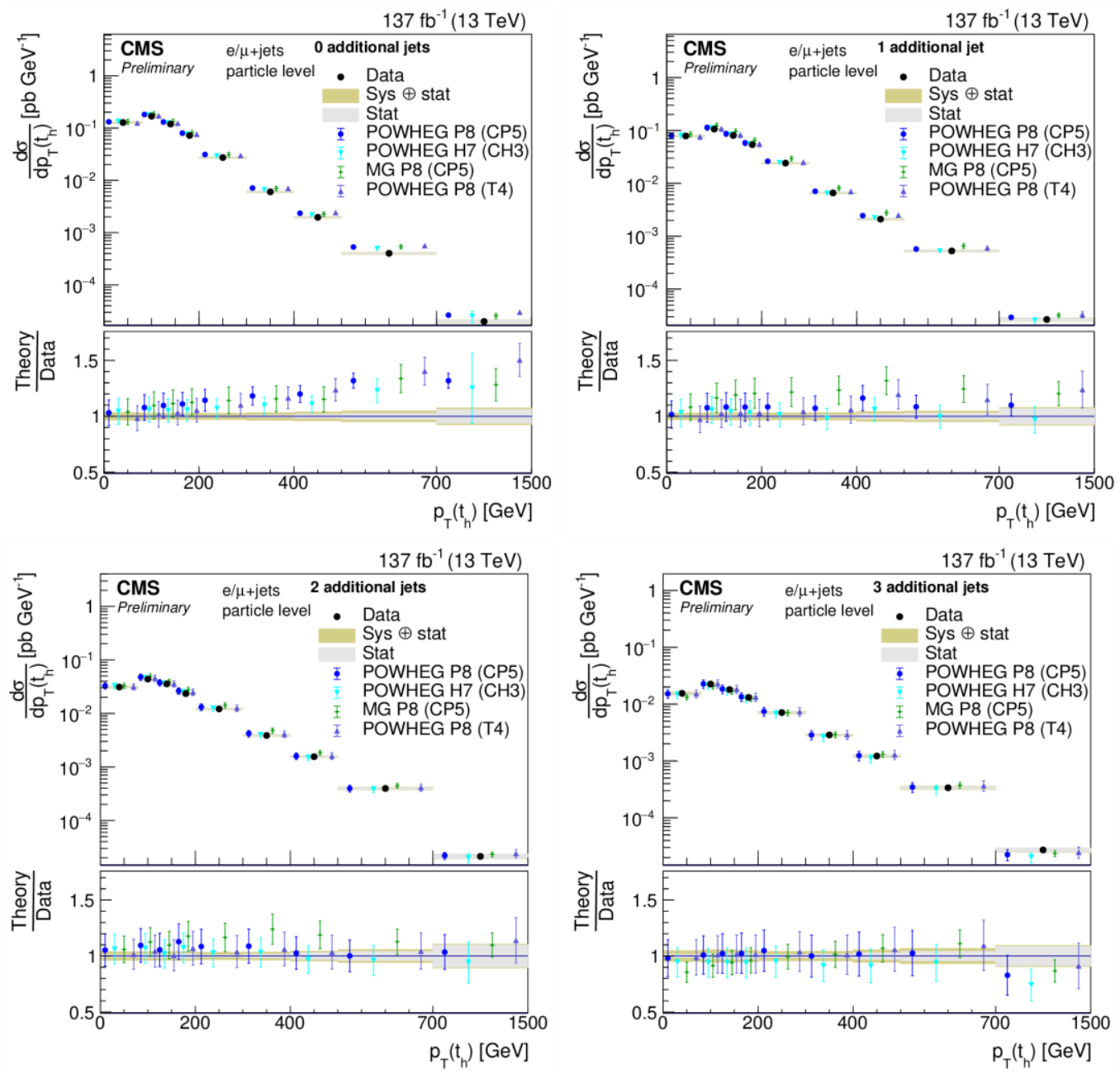

Figure 6. Differential cross sections at the particle level as a function of $\mathrm{p}_{\mathrm{T}}\left(\mathrm{t}_{\mathrm{h}}\right)$ in bins of the number of additional jets [4]. The data are shown as points with light (dark) bands indicating the statistical (statistical and systematic) uncertainties. The cross sections are compared to the predictions of POWHEG +PYTHIA (P8) for the CP5 and CUETP8M2T4 (T4), POWHEG +HERWIG (H7), and the multiparton simulation MG5_aMC@NLO (MG)+PYTHIA.

\section{References}

[1] CMS Collaboration, The CMS experiment at the CERN LHC, JINST 3 S08004 (2008)

[2] M. Czakon, P. Fiedler, A. Mitov, The total top quark pair production cross-section at hadron colliders through $O\left(\alpha_{S}^{4}\right)$, PRL 110 (2013) 252004 [arXiv:1303.6254]

[3] CMS Collaboration, Measurement of the inclusive $t \bar{t}$ cross section in pp collisions at $\sqrt{s}=5.02 \mathrm{TeV}$, CMS-PAS-TOP-20-004

[4] CMS Collaboration, Measurement of differential $t \bar{t}$ production cross sections in the full kinematic range using lepton + jets events from pp collisions at $\sqrt{s}=13 \mathrm{TeV}$, CMS-PAS-TOP-20-001

[5] CMS Collaboration, Measurement of the top quark pair production cross section in dilepton final states containing one $\tau$ lepton in pp collisions at $\sqrt{s}=13$ TeV, J. High Energ. Phys. 2020, 191 (2020)

[6] CMS Collaboration, Measurement of differential t $\bar{t}$ production cross sections using top quarks at large transverse momenta in pp collisions at $\sqrt{s}=13 T e V$, Phys. Rev. D 103, 052008 